\title{
Sífilis em gestantes residentes em São Luís, Maranhão: perfil e evolução de 2006 a 2018
}

\author{
Syphilis in pregnant women living in São Luís, Maranhão: profile and \\ evolution from 2006 to 2018
}

\section{Sífilis en gestantes residentes en São Luís, Maranhão: perfil y evolución de 2006 a 2018}

Judriele Francisca Vieira Fernandes ${ }^{1, a}$

judriellyfernandes@gmail.com | https://orcid.org/o000-0003-3397-1391

Rômulo Cesar Rezzo Pires ${ }^{2, b}$

romulo.pires@yahoo.com.br | https://orcid.org/0000-0003-0967-3351

Andréa Martins Cantanhede ${ }^{3, c}$

andrea.cantanhede@ufma.br | https://orcid.org/0000-0002-3290-0236

Enndrio Eduardo da Silva Cordeiro ${ }^{1, a}$

endo.eduardo28@gmail.com | https://orcid.org/o0o0-0001-6237-4639

\footnotetext{
${ }^{1}$ Faculdade do Maranhão. São Luís, MA, Brasil.

2 Secretaria de Estado da Educação do Maranhão. São Luís, MA, Brasil.

${ }^{3}$ Universidade Federal do Maranhão. Chapadinha, MA, Brasil.

a Graduação em Enfermagem pela Faculdade do Maranhão.

b Mestrado em Saúde e Ambiente pela Universidade Federal do Maranhão.

c Doutorado em Genética, Conservação e Biologia Evolutiva pelo Instituto Nacional de Pesquisas da Amazônia.
}

\section{RESUMO}

Realizou-se um estudo descritivo com os casos notificados de Sífilis Gestacional (SG) em São Luís (Maranhão) no período de 2006 a 2018. Foram analisadas as tendências de indicadores epidemiológicos pela regressão Joinpoint e as características do agravo com base nos dados do Sistema de Informação de Agravos de Notificação (Sinan). Foram notificados 1.688 casos de SG no período estudado, com tendência significativa de aumento no número de casos novos, na prevalência e no coeficiente de detecção. O perfil das gestantes com SG mostrou que a infecção foi mais frequente em mulheres de 20 a 29 anos, com ensino médio e pardas. Além disso, houve predomínio da forma primária, frequentemente detectada no terceiro trimestre gestacional e tratada com penicilina. A persistência da infecção por sífilis na gestação demonstra uma falha na prevenção de infecções sexuais na população, especialmente na gestação, implicando riscos para e mãe e para o concepto.

Palavras-chave: Sífilis; Gravidez; Sistema de Informação; Indicadores; Epidemiologia. 


\title{
ABSTRACT
}

A descriptive study was performed with the reported cases of Gestational Syphilis (GS) in São Luís (Maranhão) from 2006 to 2018. We analyzed the trends of epidemiological indicators by the Joinpoint regression and the characteristics of the disease based on the data from the Notifiable Disease Information System (Sinan). 1,688 cases of GS were reported in the period studied, with a significant tendency of increasing in the number of new cases, prevalence and detection coefficient. The profile of pregnant women with GS showed that the infection was more frequent in women aged 20 to 29 years, with high school and mixed race. Additionally, there was a predominance of the primary form, frequently detected in the third trimester of pregnancy and treated with penicillin. The persistence of syphilis infection during pregnancy demonstrates a failure to prevent sexual infections in the population, especially during pregnancy, implying risks for both the mother and the fetus.

Keywords: Syphilis; Pregnancy; Information system; Indicators; Epidemiology.

\section{RESUMEN}

Estudio descriptivo con los casos notificados de Sífilis Gestacional (SG) en São Luís (Maranhão) de 2006 a 2018. Se analizaron las tendencias de los indicadores epidemiológicos mediante regresión Joinpoint y las características de la enfermedad con base en datos del Sistema de Información de Quejas formales (Sinan). Se notificaron 1.688 casos de SG en el período estudiado, con una tendencia significativa al aumento en el número de nuevos casos, en la prevalencia y en el coeficiente de detección. El perfil de gestantes con SG mostró que la infección fue más frecuente en mujeres de 20 a 29 años, con estudios secundarios y mestizas. Además, predominó la forma primaria, frecuentemente detectada en el tercer trimestre del embarazo y tratada con penicilina. La persistencia de la infección por sífilis durante el embarazo demuestra la falta de prevención de las infecciones sexuales en la población, especialmente durante el embarazo, lo que implica riesgos tanto para la madre como para el feto.

Palabras clave: Sífilis; Embarazo; Sistema de información; Indicadores; Epidemiología.

\begin{abstract}
Contribuição dos autores:
Concepção e desenho do estudo: Judriele Francisca Vieira Fernandes.

Aquisição, análise ou interpretação dos dados: Judriele Francisca Vieira Fernandes, Rômulo Cesar Rezzo Pires.

Redação do manuscrito: Judriele Francisca Vieira Fernandes, Rômulo Cesar Rezzo Pires.

Revisão crítica do conteúdo intelectual: Judriele Francisca Vieira Fernandes, Rômulo Cesar Rezzo Pires, Andréa Martins

Cantanhede, Enndrio Eduardo da Silva Cordeiro.
\end{abstract}

Declaração de conflito de interesses: não há.

Fontes de financiamento: não houve.

Considerações éticas: o estudo foi submetido e aprovado pelo Comitê de Ética em Pesquisa do Instituto Florence de Ensino Superior através do Parecer $n^{\circ}$ 4.014.610 e CAAE n² 29510820.7.0000.9448.

Agradecimentos/Contribuições adicionais: não há.

Histórico do artigo: submetido: 01 set. 2020 | aceito: 18 nov. 2020 | publicado: 30 jun. 2021.

Apresentação anterior: não houve.

Licença CC BY-NC atribuição não comercial. Com essa licença é permitido acessar, baixar (download), copiar, imprimir, compartilhar, reutilizar e distribuir os artigos, desde que para uso não comercial e com a citação da fonte, conferindo os devidos créditos de autoria e menção à Reciis. Nesses casos, nenhuma permissão é necessária por parte dos autores ou dos editores. 


\section{INTRODUÇÃO}

A sífilis é uma doença infecciosa que tem um grande impacto na saúde pública (PIRES; OLIVEIRA; SOUZA; OLIVEIRA, 2013), podendo ser transmitida de forma sexual, pelo sangue e verticalmente (PINTO; TANCREDI; ALENCAR; CAMOLESI et al., 2014) a qualquer momento durante a gravidez através da placenta (SZWARCWALD; BARBOSA JUNIOR; MIRANDA; PAZ, 2007).

A Organização Mundial da Saúde (OMS) estimou para o período de 2009 a 2016 um total de 6,3 milhões de casos incidentes de sífilis, com prevalência global estimada, em homens e mulheres, de 0,5\% e valores variando de o,1 a 1,6\% nos cinco grandes continentes (ROWLEY; KORENROMP; LOW; UNEMO et al., 2019).

Estima-se que 2 milhões de gestantes estejam infectadas com sífilis, podendo resultar em transmissão vertical que ocasiona a Sífilis Congênita (SC) - cuja consequência, quando não detectada e tratada em tempo hábil na gravidez (VILIBIĆ-ČAVLEK; KOLARIĆ; PAVLIĆ; LIČINA et al., 2019), acarreta efeitos deletérios.

A morbimortalidade da sífilis congênita é elevada. Estima-se que, em 2018, 350.00o resultados adversos da gravidez em todo o mundo foram atribuídos à doença, tendo como consequência 143.000 mortes fetais/ natimortos, 62.000 óbitos neonatais, 44.000 bebês prematuros com baixo peso ao nascer e 102 mil bebês infectados (SALOOJEE; VELAPHI; GOGA; AFADAPA et al., 2004).

Os fatores associados à sífilis na gestação são: falhas no pré-natal; baixas condições socioeconômicas; dificuldades de acesso aos serviços de saúde (TEIXEIRA; SANTOS; CARVALHO; VITOR et al., 2017); além de relações sexuais de risco na adolescência (MAGALHÃES; KAWAGUCHI; DIAS; CALDERON, 2013).

Estima-se que no Brasil a taxa de detecção de SG (número de casos de sífilis gestacional por mil nascidos vivos) é da ordem de 21,4 casos por mil nascidos vivos (BOLETIM EPIDEMIOLÓGICO DE SÍFILIS, 2019). Somente no período de 2010 a 2017, essa taxa aumentou 4,9 vezes, passando de 3,5 para 17,2 casos por mil nascidos vivos (SALOOJEE; VELAPHI; GOGA; AFADAPA et al., 2004). Já a prevalência (número de casos de SG em função do total de gestações no período) encontra-se em torno de 1,4\% a 2,8\%, com transmissão vertical em aproximadamente $100 \%$ das infecções precoces e com subnotificação nacional de até $67 \%$ (CALÁS, 2015).

No período de janeiro de 2005 a junho de 2019, foram notificados no Sistema de Informação de Agravos de Notificação (Sinan) 324.321 casos de SG, dos quais 45,0\% eram residentes na região Sudeste, 21,0\% na região Nordeste, $14,7 \%$ na Sul, 10,4\% na Norte e 8,9\% na região Centro-Oeste. A partir de 2010, tem se observado aumento nas notificações, com destaque para o biênio 2017-2018, no qual o número de notificações apresentou aumento em todas as regiões, com destaque para o incremento de 59,6\% na região Nordeste (BOLETIM EPIDEMIOLÓGICO DE SÍFILIS, 2019).

Entre as capitais da região Nordeste, Recife, Salvador, Maceió, Natal, Teresina, Fortaleza e a capital do estado do Maranhão, São Luís, apresentaram as maiores taxas de detecção de SG em 2018, todas superiores à taxa nacional (21,4 casos por mil nascidos vivos) (BOLETIM EPIDEMIOLÓGICO DE SÍFILIS, 2019).

Dois estudos recentes têm destacado o aumento na incidência de casos de SG no estado do Maranhão entre os períodos de 2012 a 2017 (VERDE; OLIVEIRA; MAGALHÃES; LIMA et al., 2013) e 2009 a 2013 (GUIMARÃES; ALENCAR; FONSECA; GONÇALVES et al., 2018), neste último, o destaque foi para a capital do estado, São Luís.

Os estudos sobre SG realizados até agora no estado do Maranhão foram conduzidos em períodos curtos de tempo e não descrevem as tendências e o perfil epidemiológico da SG em São Luís, município com maior número de casos do agravo. Desse modo, este estudo verificou a tendência nos indicadores epidemiológicos de SG em São Luís e descreve o perfil das gestantes confirmadas em uma série histórica de 13 anos. 


\section{METODOLOGIA}

Realizou-se um estudo descritivo e retrospectivo com abordagem quantitativa de dados com as notificações de sífilis gestacional no período de 2006 a 2018 em São Luís, Maranhão.

São Luís, capital do Maranhão, possui população estimada em 1.101.889 habitantes e seu IDH é 0,768. O município conta com mais de 100 estabelecimentos públicos de saúde, incluindo instituições federais, estaduais e municipais de saúde. Além disso, a cidade possui também 212 estabelecimentos privados de saúde (IBGE, c2020).

Os sistemas de informação utilizados foram o Sinan, o Sistema de Informações sobre Nascidos Vivos (Sinasc), o Sistema de Informações Hospitalares (SIH) e o Sistema de Informações de Mortalidade (SIM), através da ferramenta TabNet Win do Departamento de Informática do Sistema Único de Saúde (Datasus).

O Sinan foi utilizado para acessar o número total de notificações de sífilis na gestação; o Sinasc para obter dados sobre o histórico obstétrico da mãe nos casos de nascidos vivos, além de dados dos recémnascidos; o SIM para obter dados de histórico obstétrico das mães nos casos de natimorto; e o SIH, para os casos de abortamento. Os dados foram sumarizados na Tabela 1.

Tabela 1 - Estatísticas vitais utilizadas para construção dos indicadores selecionados para o estudo

\begin{tabular}{lcccc} 
Ano & Nascidos vivos $^{\mathbf{a}}$ & Natimortos $^{\mathbf{b}}$ & Abortos $^{\mathbf{c}}$ & Total \\
\hline 2006 & 17.739 & 261 & 3.845 & 21.845 \\
2007 & 17.944 & 253 & 3.371 & 21.568 \\
2008 & 18.255 & 266 & 2.805 & 21.326 \\
2009 & 17.759 & 258 & 2.447 & 20.464 \\
2010 & 17.295 & 249 & 2.322 & 19.866 \\
2011 & 15.316 & 222 & 2.225 & 17.763 \\
2012 & 18.059 & 238 & 2.385 & 20.682 \\
2013 & 17.204 & 262 & 2.017 & 19.483 \\
2014 & 16.536 & 251 & 1.693 & 18.480 \\
2015 & 16.998 & 244 & 1.806 & 19.048 \\
2016 & 15.930 & 185 & 1.704 & 17.819 \\
2017 & 15.549 & 180 & 1.674 & 17.403 \\
2018 & 15.959 & 187 & 1.621 & 17.767 \\
Total & $\mathbf{2 2 0 . 5 4 3}$ & $\mathbf{3 . 0 5 6}$ & $\mathbf{2 9 . 9 1 5}$ & $\mathbf{2 5 3 . 5 1 4}$ \\
\hline
\end{tabular}

aSinasc, bSIM, ' $\mathrm{SIH}$

Fonte: elaboração dos autores.

A variável dependente deste estudo foi a notificação de sífilis em gestantes. Considerou-se como caso de SG o critério adotado pela Nota Informativa $\mathrm{n}^{0}$ 02-SEI/2017-DIAHV/SVS/MS, que define que todos os casos de mulheres diagnosticadas com sífilis durante o pré-natal, parto e/ou puerpério devem ser notificados como sífilis em gestantes e não como sífilis adquirida (BRASIL, 2017).

As variáveis independentes deste estudo foram: ano de diagnóstico (2006 a 2019), idade (10-14; 1519; 20-39; $\geq 40$ ), raça/cor (branca; parda; preta; amarela; indígena), escolaridade, classificação clínica, idade gestacional, esquema de tratamento e indicadores epidemiológicos (número de casos, prevalência e taxa de detecção de sífilis em gestantes). Os indicadores foram calculados até o ano de 2018, devido à indisponibilidade do número de nascidos vivos para o ano de 2019. O critério para seleção das variáveis foi a disponibilidade delas pelo Datasus. 
O Ministério da Saúde adota como indicador padrão de SG o coeficiente de detecção. Para a taxa de detecção de sífilis em gestantes (frequência anual do agravo), utilizou-se o número de casos notificados pelo número de nascidos vivos, multiplicado por 1.00o. Outro indicador adicional, que pode ser usado para fins de comparação com outras localidades, é o de prevalência de SG. Para o cálculo de prevalência da sífilis na gestação (número de casos existentes da doença em uma população) foi realizada a razão entre o número total de casos notificados de sífilis na gestação pelo número de gestações no período, multiplicado por 100, segundo o descrito por Padovani e outros especialistas (PADOVANI; OLIVEIRA; PELLOSA, 2018). O número de gestações foi obtido por meio da somatória dos nascidos vivos, abortos e natimortos registrados no período.

Foram calculadas as variações percentuais anuais (APC) para os indicadores de saúde, através da modelagem pelo método Joinpoint, usando o ano calendário como variável regressora. As análises estatísticas foram realizadas com o software Joinpoint Regression Program, versão 4.8.o.1 (Statistical Research and Applications Branch, National Cancer Institute, Bethesda, Estados Unidos).

O modelo de regressão Joinpoint é um método que permite analisar tendências temporais, avaliando se, em alguns pontos (joinpoints), existem alterações do padrão de tendência observado. O Joinpoint, que utiliza como variável dependente uma transformação logarítmica da taxa, é usado para encontrar o melhor modelo; ele testa se vários segmentos de reta (com vários joinpoints) explicam melhor uma tendência no tempo do que uma reta única. O Joinpoint utiliza testes de permutação de Monte Carlo para comparar os diversos modelos (com o, 1, 2, 3, 4 ou 5 joinpoints - a depender do tamanho da série temporal) e avaliar qual é o melhor (KIM; FAY; FEUER; MIDTHUNE, 2000).

Uma vez que o modelo é definido, a Variação Percentual Anual (APC) é calculada, para cada segmento, e usada não só para descrever e quantificar a tendência, mas também para avaliar se essa tendência é estatisticamente significativa. Nesse caso, a hipótese nula é $\mathrm{APC}=0$, isto é, as taxas não estão nem aumentando, nem diminuindo. No caso de série com presença de pelo menos 1 joinpoint, estimou-se a Variação Percentual Anual Média (APPC - mudança percentual anual média), que é calculada com base na média geométrica acumulada das tendências da APC, com pesos iguais para os comprimentos de cada segmento durante o intervalo fixado.

Uma vez que foram utilizados dados agregados, não houve manipulação de dados nominais.

\section{RESULTADOS}

Foram notificados 1.688 casos de sífilis gestacional no período de 2006 a 2019 na capital maranhense, com destaque para o ano de 2018 que concentrou 23,93\% (n=404) da casuística. $\mathrm{O}$ ano com menor número de notificações foi 2009 ( $n=13)$.

O perfil das gestantes com SG mostrou que a infecção foi mais frequente em mulheres de 20 a 29 anos (54,1\% / 4,14 por 1.000 nascidos vivos), com ensino médio (51,6\% / 3,82 por 1.0oo nascidos vivos) e pardas (85,6\% / 6,55 por 1.000 nascidos vivos) (Tabela 2). A idade média das mulheres com SG foi 25,46 7,12 anos. 
Tabela 2 - Perfil sociodemográfico de 1.688 mulheres notificadas com sifilis gestacional no período de 2006 a 2019, em São Luís, Maranhão

\begin{tabular}{lccc} 
Variável & $\mathbf{n}$ & $\%$ & $\begin{array}{c}\text { Coeficiente de } \\
\text { deteç̧ão }\end{array}$ \\
$\begin{array}{l}\text { Faixa etária (idade) } \\
\leq 14\end{array}$ & 20 & 1,2 & 0,09 \\
15 a 19 & 348 & 20,6 & 1,58 \\
20 a 29 & 913 & 54,1 & 4,14 \\
30 a 39 & 366 & 21,7 & 1,66 \\
$40+$ & 41 & 2,4 & 0,18 \\
Escolaridade & & & \\
Analfabeta & 04 & 0,2 & 0,02 \\
Fundamental incompleto & 246 & 28,1 & 1,11 \\
Fundamental completo & 134 & 8,2 & 0,61 \\
Médio incompleto & 234 & 14,3 & 1,06 \\
$\quad$ Médio completo & 608 & 37,3 & 2,76 \\
Superior incompleto & 16 & 1,0 & 0,07 \\
Superior completo & 11 & 0,7 & 0,05 \\
Ignorada & 378 & 7,5 & 1,71 \\
Raça/cora & & & \\
Branca & 81 & 4,8 & 0,37 \\
Preta & 99 & 5,9 & 0,45 \\
Amarela & 03 & 0,2 & 0,01 \\
Parda & 1.445 & 85,6 & 6,55 \\
Indígena & 06 & 0,4 & 0,03 \\
Ignorada & 54 & 3,2 & 0,24 \\
\hline
\end{tabular}

aDados disponíveis de 2006 a 2019; 'Dados disponíveis de 2007 a 2019.

Fonte: elaboração dos autores.

Com relação à SG, houve predomínio da forma primária (51,3\% / 3,79 por 1.000 nascidos vivos), frequentemente detectada no terceiro trimestre gestacional $(45,1 \%$ / 3,33 por 1.000 nascidos vivos) e tratada com penicilina (39,1\%/ o,43 por 1.000 nascidos vivos). Ainda com relação ao tratamento, destacase que em quase metade dos casos o esquema de tratamento é ignorado (47,7\% / 0,52 por 1.000 nascidos vivos) (Tabela 3). Observou-se que as taxas mais elevadas de detecção da doença coincidem com as maiores proporções, quando se compara a ocorrência da SG segundo as características maternas (Tabelas 2 e 3).

Tabela 3 - Perfil clínico de 1.688 mulheres notificadas com sífilis gestacional no período de 2006 a 2019, em São Luís, Maranhão

(continua)

\begin{tabular}{|c|c|c|c|}
\hline Variável & $\mathbf{n}$ & $\%$ & $\begin{array}{l}\text { Coeficiente de } \\
\text { detecção }\end{array}$ \\
\hline \multicolumn{4}{|c|}{ Forma clínica da doença } \\
\hline Primária & 837 & 51,3 & 3,79 \\
\hline Secundária & 221 & 13,5 & 1,00 \\
\hline Terciária & 92 & 5,6 & 0,04 \\
\hline Latente & 183 & 11,2 & 0,83 \\
\hline Ignorada & 298 & 18,3 & 1,35 \\
\hline \multicolumn{4}{|c|}{ Idade gestacional (trimestre) } \\
\hline 10 & 314 & 19,3 & 1,42 \\
\hline $2^{0}$ & 398 & 24,4 & 1,80 \\
\hline 30 & 735 & 45,1 & 3,33 \\
\hline Ignorada & 184 & 11,3 & 0,83 \\
\hline
\end{tabular}


(conclusão)

\begin{tabular}{cccc} 
Variável & $\mathbf{n}$ & $\%$ & $\begin{array}{c}\text { Coeficiente de } \\
\text { detecção }\end{array}$ \\
$\begin{array}{c}\text { Esquema de tratamentoc } \\
\text { Penicilina }\end{array}$ & 95 & 39,1 & 0,43 \\
Outro & 20 & 8,2 & 0,09 \\
Não realizado & 12 & 4,9 & 0,05 \\
Ignorado & 115 & 47,7 & 0,52 \\
\hline
\end{tabular}

bDados disponíveis de 2007 a 2019; 'Dados disponíveis de 2015 a 2018.

Fonte: elaborado pelos autores.

Durante a série estudada, houve tendência de aumento significativo no número de casos de SG com Variação Percentual Anual de 28,68\% (Tabela 4 e Figura 1).

A prevalência geral de SG foi $0,61 \%$, variando de $0,06 \%$ (2009) a 2,27\% (2018). Houve aumento significativo na prevalência de SG no período estudado com Variação Percentual Anual de 15,45\% (Tabela 4).

A taxa de detecção de SG foi 6,93 por mil nascidos vivos, variando de 0,73 (2009) a 25,31 por mil nascidos vivos (2018). Para esse indicador houve formação de um ponto de inflexão. Entre 2006 e 2010, a taxa de detecção diminuiu de forma pouco expressiva e, a partir deste ano, aumentou de maneira significante até 2018, com Variação Percentual Anual de 51,52\%. Considerando a série completa, a Variação Percentual Anual Ponderada (APPC) foi de 26,10\% (Tabela 4).

O período de maior aumento percentual de SG na série estuda foi o biênio 2017-2018, no qual o número de casos aumentou em 73,39\%, a prevalência em $69,40 \%$ e a taxa de detecção (por mil nascidos vivos) em $68,96 \%$.

Ao longo da série estudada, o número de casos de SG aumentou 16,83 vezes; a prevalência 21 vezes; e a taxa de deteç̧ão 18,75 vezes, sendo que os maiores aumentos correspondem ao biênio 2017-2018, como destacamos, com valores maiores de 1,69 vezes para prevalência; 1,73 vezes para número de casos; e 1,69 vezes para a taxa de detecção.

Tabela 4 - Indicadores epidemiológicos da sífilis gestacional no período de 2006 a 2018, em São Luís, Maranhão

\begin{tabular}{lllllll} 
Indicador & Joinpoints & Anos & APC & IC $_{95 \%}$ & Tendência & $\boldsymbol{p}$ \\
Casos notificados & 0 & 2006 a 2018 & $28,68^{*}$ & {$[16,2 ; 42,5]$} & Crescente & 0,0 \\
Prevalência (\%) & 1 & 2006 a 2018 & $15,45^{*}$ & {$[09,6 ; 21,3]$} & Crescente & 0,0 \\
Taxa de deteç̧ão & 1 & 2006 a 2010 & $-12,61$ & {$[-12,6 ; 41,9]$} & Decrescente & 0,5 \\
(por 1.000 NV) & & 2010 a 2018 & $51,52^{*}$ & {$[28,2 ; 79,1]$} & Crescente & 0,0 \\
& & & APPC & & \\
& & & $26,1^{*}$ & {$[10,4 ; 35,3]$} & Crescente & 0,0 \\
\hline
\end{tabular}

APC - Variação Percentual Anual. APPC - Variação Percentual Anual Ponderada. IC ${ }_{95 \%}$ - Intervalo de Confiança de $95 \%$. $p$ - valor de $p$ pelo teste de permutação de Monte Carlo.

Fonte: elaboração dos autores. 


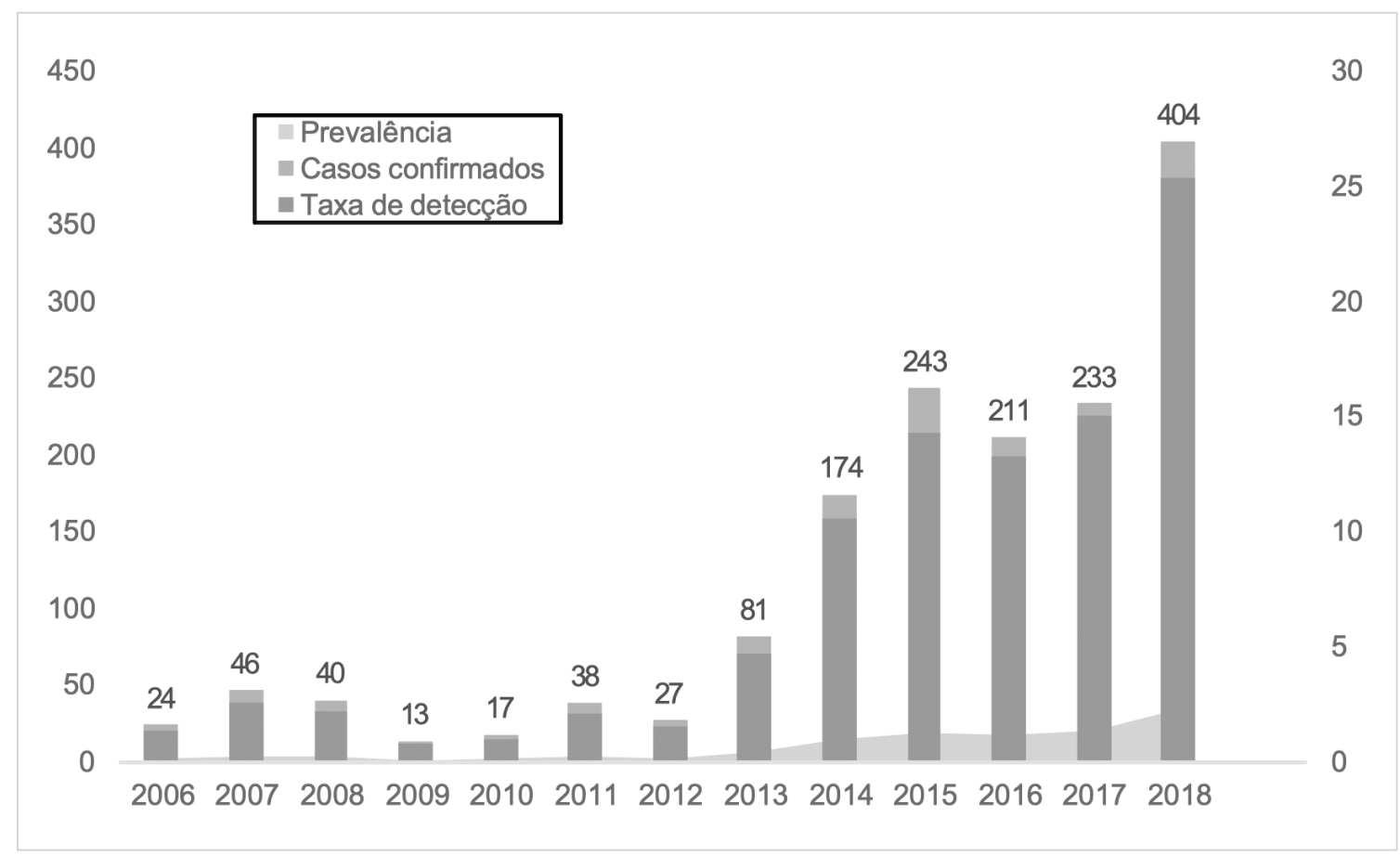

Figura 1 - Evolução dos indicadores epidemiológicos de sífilis gestacional no período de 2006 a 2018, em São Luís, Maranhão

Fonte: elaboração dos autores.

\section{DISCUSSÃO}

A sífilis é uma doença prevalente no Brasil e no mundo, persistindo como problema de saúde pública. Estima-se que aproximadamente 2 milhões, dos 11 milhões de casos novos de sífilis, ocorram em gestantes, aumentando em 50\% o risco da ocorrência de sífilis congênita (MACÊDO; LIRA; FRIAS; ROMAGUERA et al., 2017; COOPER; MICHELOW; WOZNIAK; SÁNCHEZ, 2016).

No presente estudo, as gestantes infectadas com sífilis eram adultas jovens com baixa escolaridade e pardas. A prevalência da infecção em mulheres entre 20 e 29 anos é similar aos resultados encontrados nos estudos feitos por Santos e outros pesquisadores, na cidade de Santa Maria (RS) (SANTOS; LOCK; LNZA; SANTOS et al., 2016), e por Thaíse Almeida Guimarães e outros estudiosos, no estado do Maranhão (GUIMARÃES; ALENCAR; FONSECA; GONÇALVES et al., 2018).

Destaca-se ainda a elevada prevalência de SG em mulheres de 15 a 19 anos. Isso se deve ao início da vida sexual, muitas vezes precoce e sem a devida proteção. Costa e outros (COSTA; FREITAS; SOUSA; OLIVEIRA et al., 2013) destacam a necessidade de divulgação e informação sobre as práticas sexuais no início da vida sexual. Levar informações aos adolescentes sobre a prática de sexo segura é de extrema importância para se evitar o contágio por Infecções Sexualmente Transmissíveis (IST).

Ao analisar a variável raça/cor, percebe-se a predominância de gestantes pardas e negras na cidade de São Luís. No levantamento realizado por Marques e outros estudiosos (2018), a maiorias dos casos também ocorre em pessoas pardas (80,3\%), seguidas por pessoas brancas (9,7\%) (MARQUES; ALVES; MARQUES; ARCANJO et al., 2018).

A maioria dos estudos revelam a vulnerabilidade social em relação à raça e comprovam a desvantagem de mulheres pardas e negras em conseguir uma assistência à saúde adequada. De forma geral, essas mulheres não possuem acesso a um pré-natal adequado e a uma assistência adequada no momento do parto, ou seja, elas acabam sendo vítimas dos sistemas de saúde (LEAL; GAMA; PEREIRA; PACHECO et al., 2017). 
Esse problema associa-se às divergências socioeconômicas existentes no país, no qual podemos observar indicadores baixos de acesso à saúde para pessoas pretas ou pardas (SANTOS, 2011).

Quanto à escolaridade, o mesmo perfil foi descrito em estudos realizados na Bahia (OLIVEIRA e SANTOS, 2015) e no Rio Grande do Norte (CAMPOS; ARAÚJO; MELO; ANDRADE et al., 2012). Os autores, em ambos os estudos, associam a baixa escolaridade ao crescimento dos casos de sífilis.

Com um nível de instrução menor, torna-se evidente a falta de informação acerca da prevenção de IST e sobre como manter uma vida sexual segura (COSTA; FREITAS; SOUSA; OLIVEIRA et al., 2013). Assim, a falta de instrução pode ser encarada como uma falta de conhecimento em saúde, tanto por não existir uma prevenção quanto por não ser realizado um pré-natal durante a gestação (PAIVA; CALAZANS; VENTURI; DIAS, 2008).

A forma clínica primária predominou no estudo que juntamente com os casos ignorados foram responsáveis por cerca de $70 \%$ das ocorrências. O predomínio da forma primária da doença também foi descrito no estudo de Padovani (PADOVANI; OLIVEIRA; PELLOSO, 2018).

Ter o conhecimento da caracterização clínica da sífilis é importante para que se adote um tratamento adequado para a enfermidade. Na sífilis primária, o tratamento deve envolver uma dose única semanal, enquanto que nos outros tipos, principalmente nas formas terciária e latente, as doses são maiores - com isso há uma grande taxa de abandono e a consequente complexidade do tratamento (FREITAS; MENKLE; RIVOIRE, 2011). Para além dessas questões, existe uma dificuldade em diagnosticar clinicamente a sífilis em mulheres grávidas, uma vez que o cancro duro não provoca sintomas e normalmente fica em locais onde a visualização torna-se limitada - como a parede vaginal, o períneo ou a cérvix (BRASIL, 2015).

Dessa forma, ressalta-se que possíveis erros de classificação podem levar a tratamentos inadequados, e que, na ausência de sinais clínicos e na impossibilidade de estabelecer a evolução da doença, a classificação adequada é sífilis de duração ignorada, sendo preconizado o tratamento com três doses de penicilina benzatina.

Ainda sobre a forma clínica da doença ser predominantemente a de sífilis primária, a identificação clínica da doença torna-se importante para que se avalie os riscos de transmissão vertical, que é maior na sífilis primária e secundária (BRASIL, 2017).

A contaminação do feto (transmissão vertical) pode ocorrer em qualquer período da gestação. Segundo o Ministério da Saúde, a taxa de transmissibilidade é elevada - de 70\% a 100\% na fase primária. Nessa fase da doença, a taxa de espiroquetas é elevada, fator determinante para a contaminação do feto (BRASIL, 2010).

Quase metade dos casos foi diagnosticado tardiamente (no terceiro trimestre). Os dados encontrados neste estudo corroboram com os de Souza e demais pesquisadores (2018), mas diferem dos achados de Maschio-Lima e colaboradores - autores de um estudo realizado no interior de São Paulo, no qual a maioria dos casos foi identificado no primeiro trimestre gestacional (MASCHIO-LIMA; MACHADO; SIQUEIRA; ALMEIDA, 2019). Esses resultados ressaltam as diferenças regionais no momento do diagnóstico em termos de estratégias de detecção da SG.

O fato de a doença ser diagnosticada no último trimestre gestacional pode ser justificado pelo atendimento inadequado às gestantes, durante o pré-natal, ou ainda, pela não realização do acompanhamento médico ao longo da gestação. E aí torna-se evidente o diagnóstico tardio da doença na mulher gestante.

Dessa forma, enfatizando o controle da doença, torna-se necessário uma assistência pré-natal de qualidade, com uma cobertura mais ampla do diagnóstico da gestante, que garanta um diagnóstico precoce da doença, permitido, assim, que seja realizado um tratamento antes da $24^{\mathrm{a}}$ semana de gravidez, período em que o tratamento se torna mais efetivo para o feto (DOMINGUES; SZWARCWALD; SOUZA JUNIOR; LEAL, 2013). 
O uso de penicilina como tratamento ocorreu em 39\% dos casos. Por outro lado, quase metade das mulheres $(47,7 \%)$ teve a forma de tratamento ignorada pelas entidades de saúde do município. A alta taxa de tratamento "ignorado" pode estar associada ao fato de a cidade de São Luís apresentar um elevado número de notificações no último trimestre gestacional, sugerindo ainda a falta de preparo dos profissionais da saúde no manejo com os casos de gestantes com testagem positiva, ou mesmo, pela dificuldade de obter os resultados do exame em um tempo hábil (DONALÍSIO; FREIRE; MENDES, 2007).

Apesar da universalidade no tratamento com penicilina para SG, o principal efeito adverso desse medicamento é uma reação de hipersensibilidade. Embora esse efeito seja raro, existe certa resistência a esse fármaco, tanto na aplicação pelos profissionais da atenção básica quanto na aceitação pelas gestantes. Outro fator seria o tratamento inadequado da mulher e de seu parceiro ou mesmo o desabastecimento nacional da penicilina, o que ocorreu em 2014 (MORAES; MACHADO; SOUZA, 2020).

Além da alta taxa de casos ignorados, houve uma quantidade insuficiente de mulheres tratadas com penicilina.

De acordo com o Ministério da Saúde, por meio do relatório de $n^{0} 150$, publicado no ano de 2015: recomenda-se o uso de penicilina e benzatina para o tratamento de sífilis, uma vez que não foi encontrado nenhum outro método comprovado para o tratamento efetivo durante a gravidez, sendo necessário o uso desses medicamentos na atenção primária para o tratamento da sífilis gestacional (BRASIL, 2015).

Neste estudo, verificou-se também as tendências de indicadores epidemiológicos da SG em um período retrospectivo de 13 anos. Na série estudada, todos os indicadores avaliados apresentaram tendência significativa de aumento. A notificação da SG em todo território nacional foi instituída mediante a Portaria $n^{0} 33$ de 14 de julho de 2005 (BRASIL, 2005). Neste ano de 2005, não houve notificação de SG em São Luís. A partir de 2006, o número de casos aumentou de forma significativa até 2019, com maior aumento entre 2017 e 2018, como ressaltado anteriormente.

No Brasil, considerando-se o período a partir de 2005, foram notificados no Sinan, 324.321 casos de SG, sendo que de 2017 para 2018 o número de notificações aumentou em todas as regiões do país (BOLETIM EPIDEMIOLÓGICO DE SÍFILIS, 2019). Entre os fatores associados ao incremento de casos no biênio 20172018, destaca-se o novo critério adotado para a definição de casos de SG na Nota Informativa ${ }^{0} 02$ de 19 de setembro de 2017, que considera a notificação durante o pré-natal, parto e puerpério, aumentando, assim, a sensibilidade para fins de vigilância, diminuindo a subnotificação e consequentemente levando a um aumento no número de casos.

A prevalência geral de SG foi o,61\% ao longo do período de estudo, com tendência de aumento de 15,45\%. Este valor foi inferior àqueles encontrados no Estudo Sentinela-Parturiente (1,10\%) (SZWARCWALD; BARBOSA JUNIOR; MIRANDA; PAZ, 2007) e no Estudo Nascer no Brasil (1,02\%) (DOMINGUES; SZWARCWALD; SOUZA JUNIOR; LEAL, 2014), no Rio de Janeiro-RJ (1,90\%) (DOMINGUES; SARACEN; HARTZ; LEAL, 2013) e em Belo Horizonte-MG (1,60\%) (NONATO et al., 2015). Entretanto, é superior àquele encontrado em Vitória-ES (o,4\%) (MIRANDA; ROSETTI FILHO; TRINDADE; GOUVÊA et al., 2009), em Itajaí-SC (o,43\%) (KUPEK; OLIVEIRA, 2012) e em uma regional do estado do Paraná (o,57\%) (PADOVANI; OLIVEIRA; PELLOSO, 2018).

Ao se considerar apenas o ano de 2018, verificou-se uma prevalência pontual de SG elevada (2,27\%), especialmente quando se leva em conta que uma revisão sistemática com oito estudos sobre a prevalência de SG no Brasil, com período de abrangência de 2006 a 2013, mostrou valores entre 1,02\% e 1,90\% (DANTAS; BARROS; MAIA FILHO; PARANHOS et al., 2018).

Muitos fatores podem interferir nas estimativas de prevalência da SG, entre os quais pode-se destacar: a base do estudo (nacional, estadual, municipal); a definição de caso adotada pelos autores; a fonte dos dados (agregada/ individual); o tipo de delineamento epidemiológico; a qualidade da notificação das 
estatísticas vitais utilizadas na construção do indicador (nascidos vivos e mortos); o nível de subnotificação; e a cobertura dos serviços de saúde. Além desses fatores, pode-se destacar também a limitação inerente ao intervalo de tempo e ao método utilizado para estimar a prevalência de SG.

De qualquer forma, a SG persiste como agravo prevalente e com a tendência de aumento entre as gestantes. Além disso, o indicador de prevalência é sensível ao número de gestações, valor que diminuiu em 10\% ao longo da série estudada.

A taxa geral de detecção de SG foi baixa considerando-se a série histórica completa (6,93 por mil nascidos vivos). Entretanto, a partir de 2010, seu valor apresentou tendência de aumento significativo, tornando-se, a partir de 2013, superior à taxa do estado do Maranhão; superior à taxa da região Nordeste, a partir de 2014; e à taxa nacional, do Brasil, em 2018 (BOLETIM EPIDEMIOLÓGICO DE SÍFILIS, 2019).

Além de São Luís, outras capitais brasileiras, como Rio Branco, Rio de Janeiro, Campo Grande, Vitória, Recife, Salvador, Porto Alegre, Florianópolis, Palmas, Manaus, São Paulo, Maceió, Natal, Teresina, Fortaleza, Belo Horizonte e Boa Vista apresentaram taxas elevadas de detecção de sífilis em gestantes em 2018, todas superiores à taxa nacional - o que demonstra o status da SG como problema de saúde pública reemergente (BOLETIM EPIDEMIOLÓGICO DE SÍFILIS, 2019).

Em Pernambuco, observou-se um aumento nos coeficientes de deteç̧ão de SG, especialmente em adolescentes, de cerca de 71 casos (2,1 por mil nascidos vivos) em 2007 para 289 (10,7 por mil nascidos vivos) em 2016 (OLIVEIRA; PEIXOTO; CARDOSO, 2019).

No município do Rio de Janeiro, os coeficientes de detecção de SG em adolescentes também foram superiores aos em mulheres adultas: 22,0 e 11,4 casos por mil nascidos vivos. No estado do Rio de Janeiro, foram encontrados coeficientes de 12,1 e 6,9 casos por mil nascidos vivos em adolescentes e adultas respectivamente. A comparação entre o período de 2007-2010 e 2011-2014 revelou tendência de aumento para todas as faixas etárias, sendo maior para a das adolescentes (PAGANI, 2017).

Valores elevados nas taxas de deteç̧ão podem ainda estar subestimados, uma vez que no Brasil, até o ano de 2010, a notificação atingia cerca de $32 \%$ dos casos de sífilis no período gestacional (CAMPOS; ARAÚJO; MELO; GONÇALVES, 2010) e atualmente, com a melhoria da vigilância das IST, esse valor foi estimado em 6,5\% (LAFETA; MARTELLI JUNIOR; SILVEIRA; PARANAIBA, 2016). Sendo assim, os resultados encontrados neste estudo em relação aos indicadores epidemiológicos de SG apontam para deficiências em ações efetivas de prevenção e controle desse agravo, especialmente considerando sua fácil detecção e tratamento disponibilizado pelo SUS, sendo portanto evitável (SALOOJEE; VELAPHI; GOGA; AFADAPA et al., 2004).

Muitos fatores podem estar envolvidos no aumento progressivo das taxas de detecção de SG. Fatores como a melhoria na vigilância sanitária, a ampliação da cobertura de acompanhamento do pré-natal e a implantação de programas governamentais, como Estratégia Saúde da Família (ESF), que realiza o primeiro contato e presta assistência às grávidas, são ações que contribuem, sem dúvida, para o aumento da detecção da doença (COSTA; FREITAS; SOUSA; OLIVEIRA et al., 2013).

Um estudo conduzido em 30 municípios do Maranhão mostrou que 46,8\% relataram a realização de seis consultas ou mais de pré-natal, durante a última gravidez; e que $64,6 \%$ iniciaram o pré-natal ainda no primeiro trimestre. A cobertura do pré-natal nesses 30 municípios maranhenses foi de $85,6 \%$, embora a média nacional preconizada pelo Ministério da Saúde, com relação à cobertura de pré-natal adequada, foi da ordem de 43,4\%. (COSTA; CHIEN; GAMA; COELHO et al., 2010).

Outro aspecto a ser considerado é o fato de São Luís ser a capital do e estado do Maranhão. De um modo geral, as doenças infecciosas têm maior probabilidade de se disseminar em municípios maiores e com maior densidade demográfica, como é o caso de São Luís (ROCHA; THORSON; LAMBIOTTE, 2015). Soma-se a 
este fato uma melhor infraestrutura de saúde para detecção e tratamento dos casos, o que impacta na notificação dos casos.

Segundo o Boletim Epidemiológico da Sífilis e o Plano Estadual de Saúde (PES) no Maranhão, o aumento de casos pode ser atribuído, em parte, à ampliação da cobertura de testagem, à difusão, mas, também, descentralização do uso de testes rápidos, à redução do uso de preservativo e ao desabastecimento mundial de penicilina. Além disso, o aprimoramento do sistema de vigilância pode se refletir no aumento de casos notificados (BOLETIM EPIDEMIOLÓGICO SÍFILIS, 2018).

Outro aspecto a ser considerado é a implementação de políticas de saúde materno-infantil, como a Rede Cegonha que foi instituída em 2011. Um estudo conduzido no estado do Maranhão entre 2007 e 2018 mostrou que houve redução dos casos de sífilis em sua forma mais tardia (latente e terciária). Além disso, o esquema de tratamento materno se manteve estacionário em todos os indicadores: adequado, inadequado e não realizado. Entretanto, o indicador não realizado apresentou ponto de inflexão, com mudança de tendência a partir de 2010 (BRASIL, 2005).

Com relação aos fatores associados à prevenção e ao controle da SG, uma revisão sistemática que incluiu 24 estudos epidemiológicos, 6 deles conduzidos no Brasil, mostrou que: gestantes interrompem o cuidado pré-natal precocemente e não conseguem realizar os procedimentos de triagem para sífilis; são necessárias melhorias na rede de apoio diagnóstico para enfrentamento da sífilis na gestação, no manejo clínico da doença e na investigação dos casos como evento sentinela da qualidade da assistência pré-natal; e, por fim, que a falta de tratamento adequado dos casos de sífilis em gestantes pode estar associada à morbimortalidade dos conceptos - o que mantém essa infecção como um fardo no rol dos problemas de saúde pública (OLIVEIRA; CANINI; MASIERO; SILVA, 2020).

O estudo apresenta limitações inerentes ao método da utilização de dados secundários e agregados, o que dificulta o estabelecimento de relação causal. O número de variáveis disponibilizados pelo Sinan para SG a partir de 2020 limitou-se àquelas utilizadas neste estudo, o que dificulta uma melhor compreensão da dinâmica da doença no local de estudo.

Alguns indicadores não puderam ser construídos para o ano de 2019, uma vez que dependem do número de nascidos vivos, estatística que não está disponibilizada pelo Sinasc para o referido ano. Além disso, os dados de 2019 para SG ainda estão susceptíveis a revisões e atualizações.

Apesar das limitações encontradas, o estudo forneceu um panorama de 13 anos sobre a SG, considerando que a notificação compulsória desse agravo teve início no ano de 2005 e mostrou tendências preocupantes baseadas em modelos robustos de regressão.

\section{CONCLUSÃO}

Durante o período estudado, houve aumento nas notificações de sífilis na gestação na capital do estado do Maranhão, São Luís, especialmente nos últimos anos da série histórica. Esse crescimento influenciou a tendência de aumento dos indicadores selecionados para o estudo e repercutiu na melhoria do sistema de notificação; por outro lado, falhas na detecção e no tratamento precoces fazem parte desse cenário.

O perfil encontrado foi similar ao da literatura nacional, no qual a infecção foi mais frequente em mulheres de 20 a 29 anos, com ensino médio e pardas. Além disso, houve predomínio da forma primária da doença, frequentemente detectada no terceiro trimestre gestacional e tratada com penicilina. A persistência da infecção por sífilis na gestação demonstra a fragilidade dos serviços de saúde na detecção e no tratamento precoce desse agravo. 
Reciis - Revista Eletrônica de Comunicação, Informação \& Inovação em Saúde, Rio de Janeiro, v. 15, n. 2, p. 362-378, abr./jun. 2021 [www.reciis.icict.fiocruz.br] e-ISSN 1981-6278

\section{REFERÊNCIAS}

BOLETIM EPIDEMIOLÓGICO DE SíFILIS. Brasília, DF: Ministério da Saúde, a. 5, n. 1, out. 2019. Número Especial. Disponível em: http://www.aids.gov.br/pt-br/pub/2019/boletim-epidemiologico-sifilis-2019. Acesso em: 31 dez. 2019.

BOLETIM EPIDEMIOLÓGICO SÍFILIS. São Luís: Secretaria de Saúde do Maranhão, ano 4, n. 1, set. 2018. Disponível em: https://www.mpma.mp.br/arquivos/COCOM/boletim_sifilis_2018_10_10.pdf. Acesso em: 03 nov. 2020.

BRASIL. Ministério da Saúde. Secretaria de Vigilância em Saúde. Portaria nº 33, de 14 de julho de 2005. Inclui doenças à relação de notificação compulsória, define agravos de notificação imediata e a relação dos resultados laboratoriais que devem ser notificados pelos Laboratórios de Referência Nacional ou Regional. Diário Oficial da União, Brasília, DF, 14 jul. 2005. Disponível em: http://bvsms.saude.gov.br/bvs/saudelegis/ svs/2005/prt0033 1407 2005.html. Acesso em: 31 dez. 2019.

BRASIL. Ministério da Saúde. Secretaria de Atenção à Saúde. Departamento de Ações Programáticas Estratégicas. Gestação de alto risco: manual técnico. 5. ed. Brasília: O Ministério, 2010. Disponível em: https://bvsms.saude.gov.br/bvs/publicacoes/gestacao alto risco.pdf. Acesso em: 9 jun. 2021.

BRASIL. Ministério da Saúde. Secretaria de Vigilância em Saúde. Departamento de DST, Aids e Hepatites Virais. Protocolo clínico e diretrizes terapêuticas para Atenção Integral às pessoas com infecções sexualmente transmissíveis. Brasília, DF: O Ministério, 2015. E-book. Disponível em: http://bvsms.saude. gov.br/bvs/publicacoes/protocolo clinico diretrizes terapeutica atencao integral pessoas infeccoes sexualmente transmissiveis.pdf. Acesso em: 31 dez. 2019.

BRASIL. Ministério da Saúde. Departamento de Doenças de Condições Crônicas e Infecções Sexualmente Transmissíveis. Nota Informativa n 02-SEI/2017-DIAHV/SVS/MS: altera os critérios de definição de casos para notificação de Sífilis Adquirida, Sífilis em Gestantes e Sífilis Congênita. Brasília, DF: O Ministério, 2017. Disponível em: http://www.aids.gov.br/pt-br/legislacao/nota-informativa-no-02-sei2017-diahvsvsms. Acesso em: 31 dez. 2019.

CALÁS, Jorge Eduardo de Souza. Sífilis Gestacional em municípios selecionados da Região Metropolitana I do estado do Rio de Janeiro, 2011 a 2013. 2015. 77 f. Dissertação (Mestrado Profissional em Saúde Pública) - Fundação Oswaldo Cruz, Rio de Janeiro. 2015. Disponível em: https://www.arca.fiocruz. br/bitstream/icict/13477/1/ve Jorge Eduardo ENSP 2015. Acesso em: 12 dez. 2019.

CAMPOS, Ana Luiza de Araújo; ARAÚJO, Maria Alix Leite; MELO, Simone Paes de; GONÇALVES, Marcelo Luiz Carvalho. Epidemiologia da Sífilis Gestacional em Fortaleza, Ceará, Brasil: um agravo sem controle. Cadernos de Saúde Pública, Rio de Janeiro, v. 26, n. 9, p. 1.747-1.755, set. 2010. DOI: https://doi. org/10.1590/S0102-311X2010000900008. Disponível em: http://www.scielo.br/scielo.php?script=sci arttext\&pid=S0102-311X2010000900008\&lng=en. Acesso em: 31 ago. 2020.

CAMPOS, Ana Luiza de Araújo; ARAÚJO, Maria Alix Leite; MELO, Simone Paes de; ANDRADE, Roumayne Fernandes Vieira et al. Sífilis em parturientes: aspectos relacionados ao parceiro sexual. Revista Brasileira de Ginecologia e Obstetrícia, Rio de Janeiro, v. 34, n. 9, p. 397-402, set. 2012. DOI: https://doi. org/10.1590/S0100-72032012000900002. Disponível em: http://www.scielo.br/scielo.php?script=sci arttext\&pid=S0100-72032012000900002\&lng=en. Acesso em: 21 mar. 2018.

COOPER, Joshua M.; MICHELOW, lan C.; WOZNIAK, Phillip S.; SÁNCHEZ, Pablo J. Em tempo: a persistência da Sífilis Congênita no Brasil - Mais avanços são necessários! Revista Paulista de Pediatria, São Paulo, v. 34, n. 3, p. 251-253, 2016. DOI: http://dx.doi.org/10.1016/j.rppede.2016.06.004. Disponível em: https://www.scielo.br/pdf/rpp/v34n3/pt_0103-0582-rpp-34-03-0251.pdf. Acesso em: 11 dez. 2019.

COSTA, Camila Chaves da; FREITAS, Lydia Vieira; SOUSA, Deise Maria do Nascimento; OLIVEIRA, Lara Leite de et al. Sífilis Congênita no Ceará: análise epidemiológica de uma década. Revista da Escola de Enfermagem da USP, São Paulo, v. 47, n. 1, p. 152-159, fev. 2013. DOI: https://doi.org/10.1590/S008062342013000100019. Disponível em: https://www.scielo.br/scielo.php?script=sci_arttext\&pid=S008062342013000100019\&Ing=en. Acesso em: 31 ago. 2020.

COSTA, Geny Rose Cardoso; CHEIN, Maria Bethânia da Costa; GAMA, Mônica Elinor Alves; COELHO, Leidyane Silva Caldas et al. Caracterização da cobertura do pré-natal no estado do Maranhão, Brasil. Revista Brasileira de Enfermagem, Brasília, DF, v. 63, n. 6, p. 1.005-1.009, nov.-dez. 2010. Disponível em: https:// www.scielo.br/pdf/reben/v63n6/21.pdf. Acesso em: 11 dez. 2019. 
DANTAS, Deborah Rose Galvão; BARROS, Hanna Sthefanie Tavares; MAIA FILHO, Lauriston Flávio dos Santos; PARANHOS, Luiza di Credico et al. Prevalence of Gestational and Congenital Syphilis in Brazil in the last 15 years. Journal of Infectious Diseases \& Preventive Medicine, [s. I.], v. 6, n. 3, p. 1-5, 2018. DOI: http://www.doi.org/10.4172/2329-8731.1000184. Disponível em: https://www.longdom.org/open-access/ prevalence-of-gestational-and-congenital-syphilis-in-brazil-in-the-last-15-years-2329-8731-1000184.pdf. Acesso em: 11 dez. 2019.

DOMINGUES, Rosa Maria Soares Madeira; SARACEN, Valeria; HARTZ, Zulmira Maria de Araújo; LEAL, Maria do Carmo. Sífilis Congênita: evento sentinela da qualidade da assistência pré-natal. Revista de Saúde Pública, São Paulo, v. 47, n. 1, p. 147-157, fev. 2013. DOI: https://doi.org/10.1590/S003489102013000100019. Disponível em: http://www.scielo.br/scielo.php?script=sci_arttext\&pid=S003489102013000100019\&lng=en. Acesso em: 20 jun. 2020.

DOMINGUES, Rosa Maria Soares Madeira; SZWARCWALD, Célia Landmann; SOUZA JUNIOR, Paulo Roberto Borges; LEAL, Maria do Carmo. Prevalence of syphilis in pregnancy and prenatal syphilis testing in Brazil: birth in Brazil study. Revista de Saúde Pública, São Paulo, v. 48, n. 5, p. 766-774, out. 2014. DOI: https://doi.org/10.1590/S0034-8910.2014048005114. Disponível em: http://www.scielo.br/scielo. php?script=sci arttext\&pid=S0034-89102014000500766\&lng=en. Acesso em: 20 jun. 2020.

DONALÍSIO, Maria Rita; FREIRE, June Barreiros; MENDES, Elisa Teixeira. Investigação da Sífilis Congênita na microrregião de Sumaré, estado de São Paulo, Brasil: desvelando a fragilidade do cuidado à mulher gestante e ao recém-nascido. Epidemiologia e Serviços de Saúde, Brasília, DF, v. 16, n. 3, p. 165-173, set. 2007. DOI: http://dx.doi.org/10.5123/S1679-49742007000300003. Disponível em: http://scielo.iec.gov.br/ scielo.php?script=sci_arttext\&pid=S1679-49742007000300003\&Ing=pt\&nrm=iso\&tlng=pt. Acesso em: 27 ago. 2019.

FREITAS, Fernando; MENKLE, Carlos Henrique; RIVOIRE, Waldemar Augusto; PASSOS, Eduardo Pandolfi. Rotinas em ginecologia. 6. ed. São Paulo: Artmed Editora, 2011.

GUIMARÃES, Thaíse Almeida; ALENCAR, Larissa Cristina Rodrigues; FONSECA, Lena Maria Barros; GONÇALVES, Monniely Mônica Costa et al. Sífilis em gestantes e Sífilis Congênita no Maranhão. Arquivos de Ciências e Saúde, São José do Rio Preto, v. 25, n. 2, p. 24-30, jul. 2018. DOI: https://doi. org/10.17696/2318-3691.25.2.2018.1023. Disponível em: http://www.cienciasdasaude.famerp.br/index. php/racs/article/view/1023. Acesso em: 9 jan. 2021.

INSTITUTO BRASILEIRO DE GEOGRAFIA E ESTATÍSTICA (IBGE). São Luís. [S. I.]: IBGE, c2020. Disponível em: https://www.ibge.gov.br/cidades-e-estados/ma/sao-luis.html. Acesso em: 11 fev. 2020.

JOINPOINT REGRESSION PROGRAM. Versão 4.8.0.1. Bethesda: National Cancer Institute, Estados Unidos, c2020. Disponível em: https://surveillance.cancer.gov/joinpoint/download. Acesso em: 9 jun. 2021.

KIM, Hyune-Ju; FAY, Michael P.; FEUER, Eric J.; MIDTHUNE, Douglas N. Permutation tests for Joinpoint regression with application to cancer rates. Statistics in Medicine, [s. I.], v. 19, n. 3, p. 335-351, jan. 2000. DOI: https://doi.org/10.1002/(SICI)1097-0258(20000215)19:3<335::AID-SIM336>3.0.CO;2-Z. Disponível em: https://onlinelibrary.wiley.com/doi/10.1002/(SICI)1097-0258(20000215)19:3\%3C335::AIDSIM336\%3E3.0.CO;2-Z. Acesso em: 11 fev. 2020.

KUPEK, Emil; OLIVEIRA, Juliana Fernandes de. Transmissão vertical do HIV, da sífilis e da hepatite B no município de maior incidência de AIDS no Brasil: um estudo populacional no período de 2002 a 2007. Revista Brasileira de Epidemiologia, São Paulo, v. 15, n. 3 p. 478-487, set. 2012. DOI: https://doi. org/10.1590/S1415-790X2012000300004. Disponível em: http://www.scielo.br/scielo.php?script=sci arttext\&pid=S1415-790X2012000300004\&Ing=en. Acesso em: 20 jun. 2020.

LAFETA, Kátia Regina Gandra; MARTELLI JUNIOR, Hercílio; SILVEIRA, Marise Fagundes; PARANAIBA, Lívia Máris Ribeiro. Sífilis materna e congênita, subnotificação e difícil controle. Revista Brasileira de Epidemiologia, São Paulo, v. 19, n. 1, p. 63-74, 2016. DOI: https://doi. org/10.1590/1980-5497201600010006. Disponível em: https://www.scielo.br/scielo.php?pid=S1415790X2016000100063\&script=sci_abstract\&tlng=pt. Acesso em: 31 out. 2020.

LEAL, Maria do Carmo; GAMA, Silvana Granado Nogueira da; PEREIRA, Ana Paula Esteves; PACHECO, Vanessa Eufrauzino et al. A cor da dor: iniquidades raciais na atenção pré-natal e ao parto no Brasil. Cadernos de Saúde Pública, Rio de Janeiro, v. 33, supl. 1, p. e00078816, jul. 2017. DOI: https://doi.org/10.1590/0102311x00078816. Disponível em: https://www.scielo.br/scielo.php?script=sci_arttext\&pid=S0102311X2017001305004\&lng=en. Acesso em: 31 ago. 2020. 
MACÊDO, Vilma Costa de; LIRA, Pedro Israel Cabral de; FRIAS, Paulo Germano de; ROMAGUERA, Luciana Maria Delgado et al. Risk factors for syphilis in women: case-control study. Revista de Saúde Pública, São Paulo, v. 51, 1-12, 2017. DOI: https://doi.org/10.11606/s1518-8787.2017051007066. Disponível em: http:// www.scielo.br/scielo.php?script=sci arttext\&pid=S0034-89102017000100268\&lng=pt. Acesso em: 31 ago. 2020.

MAGALHÃES, Daniela Mendes dos Santos; KAWAGUCHI, Inês Aparecida Laudares; DIAS, Adriano; CALDERON, Iracema de Mattos Paranhos. Sífilis materna e congênita: ainda um desafio. Cadernos de Saúde Pública, Rio de Janeiro, v. 29, n. 6, p. 1.109-1.120, jun. 2013.DOI: https://doi.org/10.1590/S0102311X2013000600008. Disponível em: http://www.scielo.br/scielo.php?script=sci_arttext\&pid=S0102311X2013000600008\&Ing=en. Acesso em: 31 ago. 2019.

MARQUES, João Vitor Souza; ALVES, Beatriz Mendes; MARQUES, Marcos Vinícius Souza; ARCANJO, Francisco Plácido Nogueira et al. Perfil epidemiológico da Sífilis Gestacional: clínica e evolução de 2012 a 2017. SANARE Revista de Políticas Públicas, Sobral, v. 17, n. 2, p. 13-20, 2018. DOI: https://doi. org/10.36925/sanare.v17i2.1257. Disponível em: https://sanare.emnuvens.com.br/sanare/article/ view/1257/0. Acesso em: 27 jul. 2019.

MASCHIO-LIMA, Taiza; MACHADO, lara Lúcia de Lima; SIQUEIRA, João Paulo Zen; ALMEIDA, Margarete Teresa Gottardo. Epidemiological profile of patients with congenital and gestational syphilis in a city in the State of São Paulo, Brazil. Revista Brasileira de Saúde Materno Infantil, Recife, v. 19, n. 4, p. 865-872, set.-dez., 2019. DOI: https://doi.org/10.1590/1806-93042019000400007. Disponível em: https://www.scielo.br/scielo. php?script=sci_arttext\&pid=S1519-38292019000400865\&lng=en. Acesso em: 8 mar. 2020.

MIRANDA, Angélica Espinosa; ROSETTI FILHO, Eduardo; TRINDADE, Célia Regina; GOUVÊA, Gabriel Moulin et al. Prevalência de sífilis e HIV utilizando testes rápidos em parturientes atendidas nas maternidades públicas de Vitória, estado do Espírito Santo. Revista da Sociedade Brasileira de Medicina Tropical, v. 42, n. 4, p. 386-391, ago. 2009. DOI: https://doi.org/10.1590/S0037-86822009000400006. Disponível em: http:// www.scielo.br/scielo.php?script=sci_arttext\&pid=S0037-86822009000400006\&lng=en. Acesso em: 20 jun. 2020.

MORAES, Bruno Quintela Souza de; MACHADO, Michael Ferreira; SOUZA, Carlos Dornels Frere de. Impacto da Rede Cegonha nas tendências de sífilis no Maranhão 2008-2017. Revista Científica do ITPAC, Araguaína, v. 13, n. 1, p. 83-90, fev. 2020. Disponível em: https://assets.unitpac.com.br/arquivos/revista/2020-1/volume13-numero-1-fevereiro-de-2020/artigo-9.pdf. Acesso em: 1 nov. 2020.

NONATO, Solange Maria; MELO, Ana Paulo Souto; GUIMARÃES, Mark Drew Crosland. Sífilis na gestação e fatores associados à Sífilis Congênita em Belo Horizonte-MG, 2010-2013. Epidemiologia e Serviços de Saúde, Brasília, DF, v. 24, n. 4, p. 681-694, dez. 2015. DOI: http://dx.doi.org/10.5123/S1679-

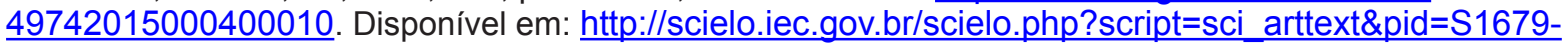
49742015000400010\&Ing=pt. Acesso em: 20 jun. 2020.

OLIVEIRA, Jamile Souza de; SANTOS, Jéssica Vasconcelos. Perfil epidemiológico da Sífilis Congênita no estado da Bahia, no período de 2010 a 2013. Revista Eletrônica Atualiza Saúde, Salvador, v. 2, n. 2, p. 20-30, jul.-dez. 2015. Disponível em: http://atualizarevista.com.br/wp-content/uploads/2015/07/Perfilepidemiol\%C3\%B3gico-da-s\%C3\%ADfilis-cong\%C3\%AAnita-no-Estado-da-Bahia-no-per\%C3\%ADodode-2010-a-2013-v.2-n.2.pdf. Acesso em: 21 mar. 2018.

OLIVEIRA, Rebeca Bezerra Bonfim de; PEIXOTO, Alisse Maria Chaves de Lima; CARDOSO, Mirian Domingos. Sífilis em gestantes adolescentes de Pernambuco. Adolescência \& Saúde, Rio de Janeiro, v. 16, n. 2, p. 47-56, abr.-jun. 2019. Disponível em: http://www.adolescenciaesaude.com/detalhe_artigo. asp?id=783. Acesso em: 27 out. 2020.

OLIVEIRA, Regiane Aparecida de; CANINI, Renata Galli; MASIERO, Anelise Viapiana; SILVA, Bruna Fernanda da. Fatores associados à prevenção e controle da Sífilis Gestacional: panorama e desafios. Contribuciones a las Ciencias Sociales, Málaga, fev. 2020. Disponível em: https://www.eumed.net/rev/cccss/2020/02/ prevencao-controle-sifilis.html. Acesso em: 02 nov. 2020.

PADOVANI, Camila; OLIVEIRA, Rosana Rosseto de; PELLOSO, Sandra Marisa. Syphilis in during pregnancy: association of maternal and perinatal characteristics in a region of southern Brazil. Revista Latino-

Americana de Enfermagem, Ribeirão Preto, v. 26, p. e3019, ago. 2018. DOI: https://doi.org/10.1590/15188345.2305.3019. Disponível em: https://www.scielo.br/scielo.php?script=sci_arttext\&pid=S0104$11692018000100335 \&$ Ing=en\&tlng=en. Acesso em: 20 jun. 2020. 
PAGANI, Marina Valverde. Sífilis Gestacional e Congênita no estado do Rio de Janeiro 2007-2014: análise comparativa entre adolescentes e adultas. 2017. 61 f. Dissertação (Mestrado em Saúde da Criança e da Mulher) - Fundação Oswaldo Cruz, Rio de Janeiro, 2017. Disponível em: https://www.arca.fiocruz.brl bitstream/icict/26358/2/marina pagani iff mest 2017.pdf. Acesso em: 9 jun. 2021.

PAIVA, Vera; CALAZANS, Gabriela; VENTURI, Gustavo; DIAS, Rita. Idade e uso de preservativo na iniciação sexual de adolescentes brasileiros. Revista de Saúde Pública, São Paulo, v. 42, supl. 1, p. 45-53, jun. 2008. DOI: https://doi.org/10.1590/S0034-89102008000800007. Disponível em: http://www.scielo.br/scielo. php?script=sci_arttext\&pid=S0034-89102008000800007\&Ing=en. Acesso em: 27 jul. 2019.

PINTO, Valdir Monteiro; TANCREDI, Mariza Vono; ALENCAR, Herculano Duarte Ramos de; CAMOLESI, Elisabeth et al. Prevalence of Syphilis and associated factors in homeless people of São Paulo, Brazil, using a Rapid Test. Revista Brasileira de Epidemiologia, São Paulo, v. 17, n. 2, p. 341-354, abr.-jun. 2014. DOI: https://doi.org/10.1590/1809-4503201400020005ENG. Disponível em: https://www.scielo.br/scielo. php?script=sci_arttext\&pid=S1415-790X2014000200341\&lng=pt. Acesso em: 21 dez. 2019.

PIRES, Muccio Costa Gondim; OLIVEIRA, Caline Novais Teixeira; SOUZA, Cláudio Lima; OLIVEIRA, Márcio Vasconcelos. Prevalence of syphilis, diagnostic methods and associated factors in patients treated in the laboratory of health Foundation of Vitória da Conquista (BA). DST - Jornal Brasileiro de Doenças Sexualmente Transmissíveis, Rio de Janeiro, v. 25, n. 4, p. 171-176, 2013. Disponível em: http://www.dst. uff.br//revista25-4-2013/DST prevalence-v25n4 IN 171-176.pdf. Acesso em: 8 dez. 2019.

ROCHA, Luis E. C.; THORSON, Anna; LAMBIOTTE, Renaud. The Non-linear health consequences of living in larger cities. Journal of Urban Health, [s. I.], v. 92, n. 5, p. 785-799. jun. 2015. DOI: http://dx.doi.org/10.1007/ s11524-015-9976-x. Disponível em: https://link.springer.com/article/10.1007/s11524-015-9976-x. Acesso em: 11 dez. 2019.

ROWLEY, Jane; HOORN, Stephen Vander; KORENROMP, Eline; LOW, Nicola; UNEMO, Magnus et al. Chlamydia, gonorrhoea, trichomoniasis and syphilis: global prevalence and incidence estimates, 2016. Bulletin of the World Health Organization, Geneva, v. 97, n. 8, p. 548-562, ago. 2019. DOI: https://dx.doi. org/10.2471\%2FBLT.18.228486. Disponível em: https://www.ncbi.nlm.nih.gov/pmc/articles/PMC6653813/. Acesso em: 1 out. 2019.

SALOOJEE, Haroon; VELAPHI, Sithembiso; GOGA, Yasmin; AFADAPA, Nike et al. The prevention and management of congenital syphilis: an overview and recomendations. Bulletin of the World Health Organization, Geneva, v. 82, n. 6, p. 424-430, jun. 2004. Disponível em: https://www.ncbi.nlm.nih.gov/pmc/ articles/PMC2622853/. Acesso em: 21 dez. 2019.

SANTOS, José Alcides Figueiredo. Desigualdade racial de saúde e contexto de classe no Brasil. Dados, Rio de Janeiro, v. 54, n. 1, p. 5-40, 2011. DOI: https://doi.org/10.1590/S0011-52582011000100001. Disponível em: https://www.scielo.br/j/dados/a/wBqxcqydPbfynmScfhHdNqH/?lang=pt. Acesso em: 9 jun. 2021.

SANTOS, Tamires Daros dos; LOCK, Nicássia Cioquetta; LNZA, Solange Schio; SANTOS, Andressa Baccin dos et al. Perfil da Sífilis Gestacional e Congênita no município de Santa Maria-RS: vivências multidisciplinares para troca de saberes. Revista Saúde (Santa Maria), Santa Maria, v. 42, n. 2, p. 215-224, jul.-dez. 2016. DOI: https://doi.org/10.5902/2236583420691. Disponível em: https://periodicos.ufsm.br/revistasaude/ article/view/20691. Acesso em: 27 jul. 2019.

SOUZA, Bárbara Soares de Oliveira; RODRIGUES, Raquel Miguel; GOMES, Raquel Maciel de Lima. Análise epidemiológica de casos notificados de sífilis. Revista da Sociedade Brasileira de Clínica Médica, v. 16, n. 2, p. 94-98, abr.-jun. 2018. Disponível em: https://docs.bvsalud.org/biblioref/2018/09/913366/16294-98.pdf. Acesso em: 27 ago. 2019.

SZWARCWALD, Célia Landmann; BARBOSA JUNIOR, Aristides; MIRANDA, Angélica E.; PAZ, Leidijany C. Resultados do Estudo Sentinela-Parturiente, 2006: desafios para o controle da Sífilis Congênita no Brasil. DST - Jornal Brasileiro de Doenças Sexualmente Transmissíveis, Rio de Janeiro, v. 19, n. 3-4, p. 128-133, 2007. Disponível em: http://www.dst.uff.br/revista19-3-2007/3.pdf. Acesso em: 15 out. 2019.

TEIXEIRA, Gracimary Alves; SANTOS, Flávia Andréia Pereira Soares dos; CARVALHO, Jovanka Bittencourt Leite de; VITOR, Allyne Fortes et al. Predisposing factors for incidence of congenital syphilis. International Archives of Medicine, [s. I.], v. 10, p. 1-8, jul. 2017. DOI: http://dx.doi.org/10.3823/2489. Disponível em: http://imedicalpublisher.com/ojs/index.php/iam/article/view/2441. Acesso em: 12 dez. 2019. 
VERDE, Roseane Mara Cardoso Lima; OLIVEIRA, Evaldo Hipólito; MAGALHÃES, Liana Moreira; LIMA, Maryana Matias Paiva de et al. Sífilis gestacional: impacto epidemiológico no estado do Maranhão,

Brasil. Research, Society and Development, São Paulo, v. 9, n. 2, p. e83922110, 2020. DOI: https://doi. org/10.33448/rsd-v9i2.2110. Disponível em: https://www.researchgate.net/publication/338318392 Sifilis gestacional_impacto_epidemiologico_no_estado_do Maranhao_Brasil. Acesso em: 11 fev. 2020.

VILIBIĆ-ČAVLEK, Tatjana; KOLARIĆ, Branko; PAVLIĆ, Jasmina; LIČINA, Mirjana Lana Kosanović et al. Seroprevalence and risk factors for HIV, hepatitis B, and syphilis in populations with high-risk behaviors in Croatia. Acta Dermatovenerologica Croatica, Zagrebe. v. 26, n. 4, p. 314-320, jan. 2019. Disponível em: https://pubmed.ncbi.nlm.nih.gov/30665481/. Acesso em: 21 dez. 2019. 